\title{
SELECTION OF TISSUE FOR MICROSCOPIC STUDY FROM LUNGS INJECTED WITH RADIO-OPAQUE MATERIAL
}

\author{
BY \\ LYNNE M. REID \\ From the Institute of Diseases of the Chest, Brompton Hospital, London
}

(RECEIVED FOR PUBlication MAY 20, 1955)

Choosing a suitable part of the lung by dissection distorts and often spoils the tissue for detailed histological study, especially when the peripheral part of the lung is concerned. These difficulties may be overcome by injecting the bronchial tree with radio-opaque material and choosing suitable areas from the abnormalities seen in a radiograph of the specimen. The parallax method is well suited for the localization of the appropriate bronchus in the depth of the lung, and its inclusion in a conveniently sized block without preliminary dissection. Trueta, Barclay, Daniel, Franklin, and Prichard (1947) give a critical survey of various injection solutions and techniques.

Method of Injection of the Bronchial Tree

Fig. 1 illustrates the apparatus used to inject the bronchial tree. A cannula was tied into the bronchus, and the lung, with the cannula protruding and open to the atmosphere, was placed in a plastic box, which could be made airtight by sealing the junction of its two component parts with vaseline. The lung was inflated by connecting a vacuum pump to the box and applying a negative pressure of $5 \mathrm{~cm}$. of mercury.

The early specimens in this series were injected with a red lead suspension, which, although satisfactorily radio-opaque, proved unsuitable as a medium for microscopic study as it set so hard that it damaged the microtome knife. For later specimens, therefore, the injection solution consisted of $70 \% \mathrm{w} / \mathrm{v}$ suspension of barium sulphate ("micropaque"-Damancy and Company, Limited) in a $7 \% \mathrm{w} / \mathrm{v}$ solution of powdered gelatine in water.

The radio-opaque solution was placed in a funnel inside a pressure cooker, which contained hot water to keep the injection solution liquid. The funnel was connected to a side opening fitted with a tap. To reduce air in the system the solution was allowed to flow to the end of the side arm before this was connected by a short piece of rubber tubing to the cannula in the lung. A pressure of $5 \mathrm{lb}$. $/ \mathrm{in}^{2}{ }^{2}$ was

\section{PRESSURE 5lb./sq. in.}

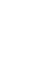


developed within the pressure cooker from a pressure pump, and then the tap was opened and the solution allowed to flow into the lung.

A whole lobe or even a lung was injected simultaneously in some specimens, while in others bronchopulmonary segments were injected separately. When injection was made in more than one stage the specimen was radiographed between each injection, since fluoroscopy is a valuable method for controlling the procedure.

This method is also satisfactory, with or without preliminary inflation, for the injection of the blood vessels.

\section{Selection of Blocks for Microscopic STUdy}

From a single view of the bronchogram suitable peripheral bronchioles were selected. These are easily visible, as they are normally of the order of $1 \mathrm{~mm}$. in diameter, and the secondary lobules they supply are about $2 \mathrm{~cm}$. in diameter. If the lesion were localized only in a single plane it would be necessary to cut the full thickness of the lung. To include it in a block without an excessive amount of surrounding tissue, it was necessary to localize it in the depth of the lung also. This was done by the method of parallax. Details of this technique have been given by Simon (1949).

The film was placed in a hollow box, open on one side and made of wood, save for the top, which was made of plastic to facilitate cleaning (Fig. 2). In this way the film was protected from moisture and could be changed without altering the position of the specimen.

The $x$-ray tube was placed 20 in. from the specimen and between the two exposures the tube was moved 4 in. parallel to the film. "Kodirex" film was used and an exposure of 1 second, 50Ma. at 40 k.v.p. The position in the depth of the lung of a particular

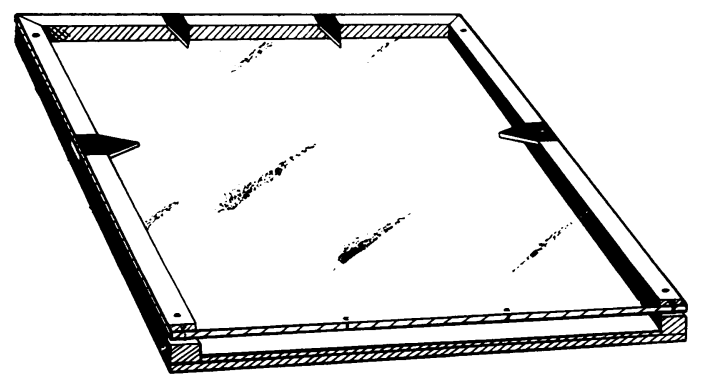

F:G. 2.-The hollow box which carries the film. It is made of wood save for the top, which is of perspex to facilitate cleaning. The open side is the lower one in the illustration. It is unnecessary to place the film in exact position in relation to the edges of the box, as the metal markers on its edge cast shadows on the film which can be used as points of comparison in serial films. Actual size is $35 \times 28 \times 2.5 \mathrm{~cm}$. shadow on the bronchogram was calculated from the formula

Distance of shadow tube-film distance $x$ image shift from film tube shift + image shift

Under the fluoroscope its position was established and $\varrho$ its relation to the surface marked with a pin. It was on thus possible to cut a block of tissue which included $\overrightarrow{0}$ the lesion without an unnecessary amount of adjacent lung.

\section{Application of the Method}

Bronchograms after injection of the lung with radio-opaque material can be used to demonstrate $\omega$ both normal and morbid anatomy. As illustra- $\vec{\theta}$ tions they are clear and detailed and can help in

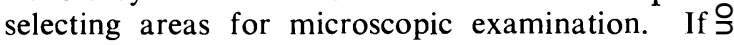
a suitable injection medium is used microscopic $\vec{c}$ study is quite satisfactory. The method described $\mathbb{\Phi}$ here of selecting and cutting blocks avoids the $\frac{\overline{0}}{\bar{\Phi}}$ necessity of identifying by dissection the structures or lesions responsible for any particular $\mathbb{\Phi}$ shadow in the radiograph. This is particularly valuable when the selected shadow is surrounded by other shadows or is in the thicker parts of the lung.

The method is well suited for showing the pathological changes which correspond to the bronchographic appearances seen in the peripheral part of the bronchial tree.

Injection of the blood vessels, both in the lungs $\overrightarrow{\vec{B}}$ and other organs, is also informative and likewise 3 proves useful in choosing parts for further examination.

\section{SUMMARY}

Injection of the bronchial tree or blood vessels in the lung with radio-opaque material aids the $\frac{0}{3}$ selection of suitable regions for histological study. An application of the parallax method is described by which the shadow in the depth of the lung can be localized. The corresponding region may then be included in a block of suitable size without the $\frac{D}{O}$ necessity of preliminary dissection.

This work was done during the tenure of a grant $\mathrm{N}$ from the Research Fund of the Hospitals for Diseases of the Chest.

\section{REFERENCES}

Simon, G. (1949), X-ray Diagnosis, p. 42. Heffer, Cambridge.

Trueta, J., Barclay, A. E., Daniel, P. M., Franklin, K. J., and Prichard, M. M. L. (1947). Studies of the Renal Circulation. Blackwell,? Oxford. 